BONPLANDIA 18(1): 45-50. 2009

\title{
TWO NEWS SPECIES OF BACCHARIS (ASTERACEAE: ASTEREAE) FROM BAHIA, BRAZIL
}

\author{
LEONARDO PAZ DEBLE ${ }^{1}$ \& ANABELA SILVEIRA DE OLIVEIRA-DEBLE ${ }^{2}$
}

\begin{abstract}
Summary: Deble, L. P. \& A. S. de Oliveira-Deble. 2009. Two new species of Baccharis (Asteraceae: Astereae) from Bahia, Brazil. Bonplandia 18(1): 45-50. ISSN: 0524-0476.

In the review of the genus Baccharis in Brazil two new species from Bahia were recognized: Baccharis alleluia and Baccharis orbiculata. These species are described, illustrated and distinguished of the allied taxa.
\end{abstract}

Key words: Baccharis, new species, Asteraceae, taxonomy, Bahia, Brazil.

Resumen: Deble, L. P. \& A. S. de Oliveira-Deble. 2009. Dos especies nuevas de Baccharis (Asteraceae: Astereae) para Bahía, Brasil. Bonplandia 18(1): 45-50. ISSN: 0524-0476.

En la revisión del género Baccharis para Brasil, fueron reconocidas dos especies nuevas: Baccharis alleluia y Baccharis orbiculata. Estas especies son descriptas, ilustradas y diferenciadas de los taxones afines.

Palabras clave: Baccharis, nuevas especies, Asteraceae, taxonomía, Bahía, Brasil.

Introduction

The genus Baccharis L. is well represented in Bahia, Hind (1993) estimated above 25-30 species, but probably a few more species occurs in this state. During the review of exsiccates, materials of two unusual species were analyzed and we recognized as new species, being described below.

\section{Baccharis alleluia Oliveira-Deble \& Deble, sp. nov.}

Fig. 1

A Baccharis salzmanii DC., primu aspectu, sed capitula 6.1-7.5 mm longa (vs. 8.5-10.4 mm longa), bracteis involucri scariosis (vs. non scariosis), corolla flores feminei 2.9-3.8 mm longa (vs. 4.4-5 mm longa), cypselis 1.1-1.5 mm longis (vs. 2.3-2.6 mm longis) et capitula in glomerulis vel corymbis longiter pedunculatis (vs. in glomerulis foliaceis, sessilis vel brevi pedunculatis), differt. Baccharis pentziifoliae Sch. Bip. ex Baker, valde proxima, a qua foliis maioribus, 2.1-6.8 cm longis, 0.9-2.8 cm latis (vs. 0.8-2.2 cm longis, 0.5-1.3 cm latis), retinervis (vs. trinervis) optime distincta. Frutex $1 \mathrm{~m}$ altus; caulibus adscendentibus erectisve, usque ad apice foliosus. Folia obovata, oblanceolata ad obdeltata, 2.6-6.5 cm longa, 1.1-2.5 cm lata, alterna, retinervia, concolora, integra vel apice utrinque 1-2 dentata, glandulosa, apice obtusa,

1 Biólogo, Dr., Departamento de Ciências Florestais, Universidade Federal de Santa Maria/RS, Brazil. deble.biol@gmail.com

2 Bióloga, Dra., Departamento de Ciências Florestais, Universidade Federal de Santa Maria/RS, Brazil. anabela.biol@gmail.com 




Fig. 1. Baccharis alleluia Oliveira-Deble \& Deble. A: female branch. B: leaves. C: female capitulum. D-F: female involucral bracts. D: outer. E: median. F: inner. G: female flower. H: cypsela. I. male capitulum. J-K: male involucral bracts. J: outer. K: median and inner. L: male flower. M: style of female flower. N: style of male flower. O: detail of apex leaf. P: apex of female flower (A-H, M, O, P, Giulietti et al. s.n. holotypus SPF; I-L, N, Sucre 10.873 paratypus $\mathrm{RB})$. 
rotunda vel truncata, base cuneata, sessilia vel brevi petiolata (petiolis usque $6 \mathrm{~mm}$ ); laminis coriaceis, castaneis ad vireo-castaneis, resinosis; Capitula sessilia vel pedunculata in glomerulis vel corymbis longiter pedunculatis ad axilam foliorum superiorum inserta. Capitula feminea cylindracea, 6.1-7.5 mm longa, 2.2-3.5 mm lata. Involucrum 5.5$6.4 \mathrm{~mm}$ longum, 1.6-2.5 mm latum, 4-5 florum. Bracteis involucri 6-7 seriati, margine scariosi, dorso straminei; externis, 0.8-2.4 mm longis, $0.7-1.1 \mathrm{~mm}$ latis; mediis lanceolatis ad elliptico-lanceolatis, 2.6$5.2 \mathrm{~mm}$ longis, 0.8-1.2 mm latis. internis linearellipticis ad linear-lanceolatis, 5.1-6.2 mm longis, 0.7-1 mm latis. Clinanthium convexum, glabrum. Flores feminei corolla tubular-filiformia, $3.2-3.6 \mathrm{~mm}$ longa, apice 5-dentata; dentibus brevi vel usque 0.3 $\mathrm{mm}$ longus. Stylo 4-4.8 mm longo; rami styli 0.7-1 $\mathrm{mm}$ longi, obtusi. Pappus stramineus ad rufescentibus, 4.1-5 mm longus; setis pappi 94-108. Cypsela 10-costata, cylindracea, 1.1-1.3 mm longa, castanea, glabra, cuticula plana.

Typus: Brazil. Bahia: Mucugê, Alto do Morro do Pina, estrada de Mucugê a Guiné, 25 km NO de Mucugê, subarbusto de $1 \mathrm{~m}$, flores creme esverdeado, odoríferas, ㅇ, 20-VII-1981, A. M. Giulietti, A. Furlan, J. Semir, I. Cordeiro, L. Rossi, J. Pirani \& N. de Menezes, s.n. (holotypus SPF 18.425!).

Shrub 0.5-2 m tall; stems erect-patent or erect, branching sympodial; young shoots brownish, glutinous, older shoots darker. Leaves ob ovate, oblanceolate to obdeltate, 2.1-6.8 cm long, 0.9-2.8 cm wide, alternate, reticulum veined, concolorous, margin entire or with 1-2 obtuse teeth near the apex, glandular, not revolute, apex obtuse, rounded or truncate, basally cuneate, sessile or shortly petiolate; leaf blades chartaceous to coriaceous, light brown to green-brownish, strongly resinous in both surfaces; petioles up to $10 \mathrm{~mm}$, light brown, glutinous. Capitulescence borne in pedicellate glomerules or corymbs inserted at the axils of the distal leaves, constituting a corymbiform panicle. Capitula sessile to pedunculate; peduncles light brown, up to $7.8 \mathrm{~mm}$ long; bracts 1-2, lanceolate, up to $3.5 \mathrm{~mm}$ long. Female capitula cylindrical, 6.1-7.5 mm long,
2.2-3.5 mm wide. Involucre 5.5-6.4 mm long, 1.6-2.5 mm wide. Involucral bracts in 6-7 series, scariose in outermost, entire or scariose only near at apex in innermost bracts, centrally stramineous and whitish in the distal third. Outer bracts ovate, 0.8-2.4 mm long, 0.7-1.1 mm wide. Median bracts lanceolate to elliptic-lanceolate, 2.6-5.2 mm long, 0.8-1.2 $\mathrm{mm}$ wide. Inner bracts linear-elliptic to linear-lanceolate, 5.1-6.2 mm long, 0.7-1 mm wide. Clinanthium slightly convex, glabrous. Female flowers 4-6. Corolla tubular-filiform, 2.9-3.8 mm long, apically 5-dentate; teeth obtuse, short or up to $0.3 \mathrm{~mm}$ long. Style exceeding the corolla, 3.7-4.8 mm long; branches 0.6-1 mm long, apex obtuse. Pappus 4.1-5 mm long; bristles 94-108. Cypsela cylindrical, 1.1-1.5 mm long, light brown, 10ribbed, glabrous, cuticle smooth. Male capitula campanulate, 4.3-5.2 mm long and wide. Involucre $3.3-4 \mathrm{~mm}$ long, 2.4-3.3 mm wide. Involucral bracts 4-5 series, scariose in outermost, entire or scariose only near at apex in innermost bracts, centrally light brown, whitish in the distal third. Outer bracts broadly ovate to ovate, $1.2-2.1 \mathrm{~mm}$ long, 1 $1.3 \mathrm{~mm}$ wide. Median bracts ovate to oblong, 2.3-3 mm long, 0.8-1.1 mm wide. Inner bracts oblongs to broadly elliptic, $2.9-3.3 \mathrm{~mm}$ long, 0.8-1 mm wide. Clinanthium convex, glabrous. Male flowers 9-15. Corolla 3-3.5 mm long; tube 1.8-2.1 mm long; lobes 1.1-1.4 $\mathrm{mm}$ long. Style not or slightly exceeding the corolla, 3.3-4 mm long; branches attached, 0.6-0.8 mm. Anthers 0.9-1.1 mm long. Pappus 2.2-3.5 mm long; bristles 43-58. Abortive cypsela obconical, $0.2-0.3 \mathrm{~mm}$ long, with short and asymmetric trichomes at apex concentrate.

Distribution and habitat: Baccharis alleluia is known only from Bahia state in Lençóis, Mucugê and Rui Barbosa cities, being found in "savanas arbustivas" or "campos rupestres" at altitudes between 900-1.100 m.

Etymology: The specific epithet is a Latin variation of the Hebrew liturgical expression "Hallelujah" significance "praise ye Yah" ("praise the Lord"), that in vernacular language meaning "happiness or happy", 
being a tribute from Bahia state, that is known as happiness or happy land.

Obs.: The sympatric species $B$. salzmanii DC. is most closely related to B. alleluia, but differs by the capitulescence borne in sessile or almost sessile glomerules, protected by distal leaves (vs. pedicellate glomerules or corymbs inserted at the axils of the distal leaves, constituting a corymbiform panicle), by capitula with 8.5-10.4 mm long (vs. 6.1$7.5 \mathrm{~mm}$ long), by margin hyaline, apex darker, not scariose involucral bracts (vs. margin scariose, apex whitish), by female corolla with 4.4-5 mm long (vs. 2.9-3.8 mm long) and by cypselas with 2.3-2.6 mm long (vs. 1.1-1.5 mm long). Another similar species is the allopatric B. pentziifolia Sch. Bip. ex Baker, but this species shows distinctly smaller, 0.8-2.2 cm long, 0.5-1.3 $\mathrm{cm}$ wide, trinerved leaves.

Paratypi: BRAZIL. Bahia: s. l., s. d., ơ , Sucre 10.873 (RB); Lençóis, Chapada Diamantina, Morro da Mãe Inácio, subarbusto $70 \mathrm{~cm}$, flores creme, 우, 18-VIII-1996, Grillo 28 \& Conceição (SPF); Rui Barbosa, savana arbustiva, planta 2 m, ox, 975 m, 16-X-1978, Faria 11 (RB).

\section{Baccharis orbiculata Deble \& Oliveira- Deble, sp. nov.}

Fig. 2

A Baccharis truncatae Gardner, cui affinis, foliis suborbiculatis ad orbiculatis (vs. obdeltatis), apice rotundatis (vs. truncatis), base rotundatis, truncatis ad cordatis (vs. cuneatis), capitulorum femineum involucris majoribus, 7.3$9.8 \mathrm{~mm}$ longum, 2.2-4.1 mm latum (vs. 4.5-5.2 mm longum, 1.8-2.4 mm latum), optime distincta. Frutex $1.8 \mathrm{~m}$ altus; caulibus adscendentibus erectisve, apice dense foliosus, postremo cicatricosis. Folia suborbiculata ad orbiculata, 10-14 mm longa, 9.7-14.5 mm lata, concolora, integerrima, conduplicata, glandulosa, alternata, apice rotundata, base rotundata, truncata vel cordata, breviter petiolata (petiolis usque 1.2 $\mathrm{mm}$ ); laminis cartaceis, resinosis, nigrescentis (in siccis). Capitula breviter pedicellata in spicis foliorum disposita. Capitula feminea cylindracea, 8.1-11.2 mm longa, 2.6-4.5 mm lata. Involucrum 7.3-9.8 mm longum, 2.2-4.1 mm latum, 6-9florum. Bracteis involucri, 6-8 seriatis, margine hyalinis, dorso castaneo, punctato-glanduloso, externis fimbriatis, intimis integris. Bracteis externis ovatis ad ovato-lanceolatis, $1.8-3.9 \mathrm{~mm}$ longis, 1.1-1.6 mm latis; mediis ovato-ellipticis ad lanceolatis, 4.2-6.3 $\mathrm{mm}$ longis, 1.2-1.5 $\mathrm{mm}$ latis; internis linear-ellipticis ad linear-lanceolatis, 6.8$9.4 \mathrm{~mm}$ longis, 1.1-1.3 mm latis. Flores feminei corolla filiformia, 4.9-5.2 $\mathrm{mm}$ longa, base bulbiformia, conspersis pilosis, apice 5-dentata; dentibus lanceolatis, 0.3-0.5 mm longis. Stylo 5.6$6.4 \mathrm{~mm}$ longo; rami styli linear-oblongi, 1-1.4 mm longi. Pappus albus, 4.2-5.4 mm longus; setis pappi 41-49. Cypsela 12-16 costata, cilyndricacea, 2.6-3.5 mm longa, glabra, cuticula striata.

Typus: Brazil. Bahia: Piatã, Catolés de Cima, próximo Rio do Bem Querer, caminho para casa do Sr. Altino $13^{\circ} 17^{\prime} \mathrm{S}, 4^{\circ}{ }^{\circ} 53^{\prime} \mathrm{W}, 1.280 \mathrm{~m}$, campo rupestre com solo argiloso e areia lavrada pelos escravos, arbusto de até $1.8 \mathrm{~m}$, folhas verde musgo, brácteas verde claro, flores creme, 우, 29-VIII-1992, W. Ganev 997 (holotypus SPF!, isotypus HUEFS).

Shrub 0.5-2.5 m tall; stems erect-patent or erect, branching sympodial; young shoots reddish brown, glutinous, older shoots darker. Leaves nearly orbicular, 8-17 mm long and wide, spirally alternate, reticulum veined, concolorous, margin entire, glandular, non revolute, apex rounded, basally rounded, truncate or cordate, shortly petiolate; leaf blades chartaceous, frequently conduplicate, dark brown, strongly resinous in both surfaces; petioles up to $1.2 \mathrm{~mm}$, reddish brown, glutinous. Capitula shortly pedunculate in leaf axils; peduncles reddish brown, up to $7.2 \mathrm{~mm}$ long; bracts $1-3$, lanceolate, up to $2.5 \mathrm{~mm}$ long. Female capitula cylindrical to broadly cylindrical 8.1-11.2 mm long, 2.6-4.5 mm wide. Involucre 7.3-9.8 $\mathrm{mm}$ long, 2.2-4.1 mm wide. Involucral bracts in 6-8 series, margin hyaline, fimbriate in outermost, very thin and entire in innermost, centrally brownish, punctate glandular and 


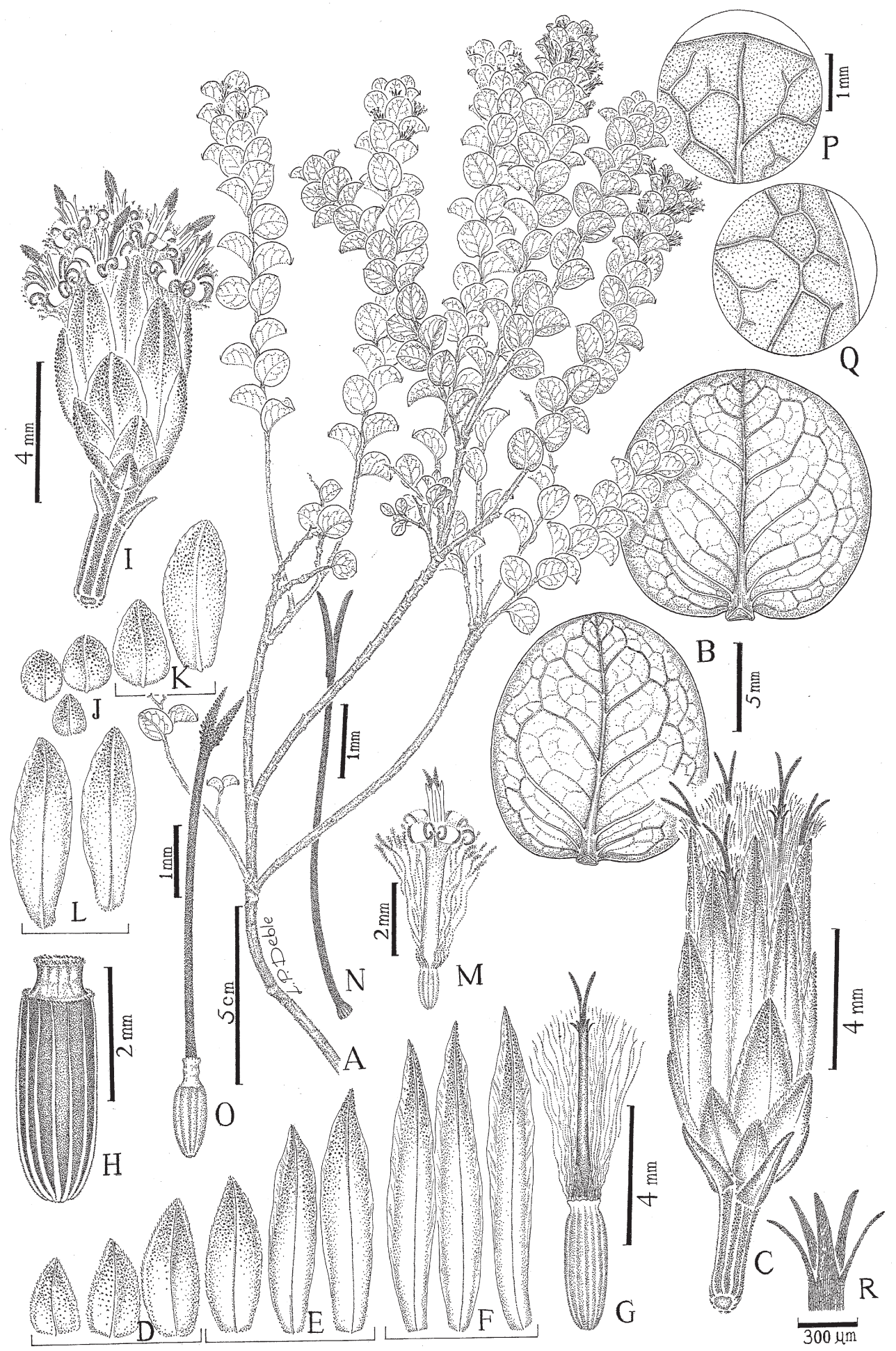

Fig. 2. Baccharis orbiculata Deble \& Oliveira-Deble. A: female branch. B: leaves. C: female capitulum. D-F: female involucral bracts. D: outer. E: median. F: inner. G: female flower. H: cypsela. I. male capitulum. J-L: male involucral bracts. J: outer. K: median. L: inner. M: male flower. N: style of female flower. O: style of male flower. P: detail of apex leaf. Q: detail of margin leaf. R: apex of female flower (A-H, N, P-R, Ganev 997 holotypus SPF; I-M, O, Ganev 734 paratypus SPF). 
darker in the distal third. Outer bracts broadly ovate to ovate-lanceolate, $1.8-3.9 \mathrm{~mm}$ long, 1.1-1.6 mm wide. Median bracts ovate-elliptic to lanceolate, $4.2-6.3 \mathrm{~mm}$ long, $1.2-1.5 \mathrm{~mm}$ wide. Inner bracts linear-elliptic to linearlanceolate, $6.8-9.4 \mathrm{~mm}$ long, $1.1-1.3 \mathrm{~mm}$ wide. Clinanthium slightly convex, glabrous. Female flowers 6-9. Corolla filiform, 4.9-5.2 $\mathrm{mm}$ long, basally bulbiform, apically 5dentate, with few trichomes at base concentrate; teeth acute $0.3-0.5 \mathrm{~mm}$ long. Style exceeding the corolla, 5.6-6.4 mm long; branches obtuse, 1-1.4 mm long. Pappus 4.2$5.4 \mathrm{~mm}$ long; bristles 41-49. Cypsela cylindrical, 2.6-3.5 mm long, light brown, 1216 ribbed, glabrous, cuticle folded. Male capitula campanulate, 6.3-9.2 mm long, 4.5-6.4 $\mathrm{mm}$ wide. Involucre 5.4-7.5 mm long, 3.8-4.9 $\mathrm{mm}$ wide. Involucral bracts 5-6 series, margin hyaline, fimbriate in outermost, very thin and entire in innermost bracts, centrally brownish, punctate glandular and darker in the distal third. Outer bracts ovate to nearly circular, 0.9-2.8 mm long, 0.8-1.7 mm wide. Median bracts broadly oblong to obovate, 3.1-4.4 mm long, 1.6-2 mm wide. Inner bracts oblongs to broadly elliptic, 4.8-6.1 mm long, 1.5-1.9 mm wide. Clinanthium convex, glabrous. Male flowers 9-16. Corolla 4.4-5.1 mm long; tube 3.2-3.6 mm long; lobes 1.2-1.5 mm long. Style slightly exceeding the corolla, 4.9-5.4 mm long; branches attached or free, 0.8-0.9 $\mathrm{mm}$. Anthers 1.2-1.4 mm long. Pappus 3.2-4 mm long; bristles 22-31. Abortive cypsela cylindrical, 0.9-1 mm long, glabrous.

Distribution and habitat: Baccharis orbiculata is known only from center of Bahia state in Abaíra, Barra da Estiva, Mucugê and Piatã cities, being fond in "campos rupestres" at altitudes between 1.100-1.300 m.

Etymology: The specific epithet refers the nearly orbicular leaves.

Obs.: Baccharis orbiculata belongs to the section Cylindricae Heering, series Axillares Giuliano, being easily distinguished of others species by nearly orbicular, frequently conduplicate, apex rounded, base rounded, truncate or cordate leaves and by the size of the female capitula. The sympatric species Baccharis truncata Gardner is related to $B$. orbiculata, but differs by its obdeltate, truncate at apex, basally cuneate leaves (vs. nearly orbicular, apex rounded, base rounded, truncate or cordate leaves), and by female involucre with 4.5-5.2 mm long, 1.8-2.4 mm wide (vs. 7.3-9.8 mm long, 2.2-4.1 mm wide). Baccharis bahiensis Baker, another similar species, differs of $B$. orbiculata by its obovate, 11-33 mm long, 7-22 mm wide leaves (vs. nearly orbicular, 8-17 mm long and wide leaves).

Paratypi: BRAZIL. Bahia: Abaíra, caminho ribeirão de Baixo-quebradas, próximo a encosta

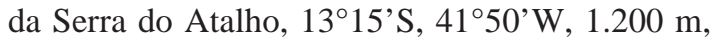
campo rupestre com solo argiloso, arbusto até 1.5 m, ơ, Ganev 794 (HUEFS, SPF!); caminho Boa

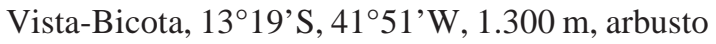
até 2.5 m, ơ', 25-VII-1992, Ganev 734 (HUEFS, SPF!); Barra da Estiva, estrada Ituaçu-Barra da Estiva, a $12 \mathrm{~km}$ de Barra da Estiva, próximo do Morro do Ouro, campo rupestre, arbusto $1.5 \mathrm{~m}, \sigma^{x}$, 17-VII-1981, Giulietti \& al. s.n. (CFCR 1.238, SPF!); Mucugê, estrada Mucugê-Guiné, 28 km de Mucugê, subarbusto 50-100 cm, ơ, 07-IX-1981, Furlan \& al. (CFCR 2027! SPF 18822!); Alto do morro do Pina, estrada de Mucugê-Guiné, a 25 km NO de Mucugê, arbusto de 1.2 m, ơ, 20-VII-1981, Giulietti \& al. s.n. (CFCR 1.538, SPF!).

\section{Acknowledgements}

The authors are indebted to the curators of SPF and RB, for making specimens available for study.

\section{Bibliography}

HIND, D. J. N. 1993. Notes on the Compositae of Bahia, Brazil: I. Kew Bull. 48 (2): 245-277.

Original recibido el 30 de junio de 2009; aceptado el 21 de julio de 2009. 